\title{
Sacroiliac joint pain: Where are we
}

\section{now?}

\section{Olena Klahsen and Stephen H.M. Brown}

Department of Human Health and Nutritional Sciences, College of Biological Science, University of Guelph, Guelph, ON Canada. For correspondence, please email: oklahsen@uoguelph.ca.

\begin{abstract}
Sacroiliac joint pain is often interpreted by healthcare practitioners as non-specific pain in the lower back region. This has resulted in many cases of sacroiliac joint pain being misdiagnosed as general chronic low back pain, which has led to large gaps in the knowledge regarding its exact causes and manifestation. The pain is theorized to develop because of the loss of form and/or force closure leading to destabilization and sacroiliac joint dysfunction. Current diagnostic tests include pain provocation tests and intra-articular injections. The intra-articular injections may exclude individuals with extra-articular pain, so more inclusive testing should be considered for use in clinical practice such as range of motion assessment or pubic symphysis analysis. Recent literature does not support the use of present treatments by healthcare practitioners as the therapies lack well designed studies to confirm their efficacy, are extremely invasive, or have minimal symptom management capabilities. Additional research into current and new treatment strategies needs to be done to determine their efficacy in managing this condition. Overall, the development of an animal model of the condition is recommended to better evaluate and improve the diagnosis and treatment of sacroiliac joint pain.
\end{abstract}

Keywords: sacroiliac joint pain, biomechanics, conservative treatment, intra-articular injections, low back pain

\section{Introduction}

The sacroiliac joint (SIJ) is made up of the articulating surfaces of the sacrum and the ilium portion of the innominate bone and is held together by a fascial network of sacroiliac ligaments (Cohen, Chen, \& Neufeld, 2013). While these ligaments are largely responsible for the stability of the pelvic ring, they are also attachment points for multiple inferior trunk and proximal lower limb muscles, such as the gluteus maximus, the erector spinae, and the biceps femoris (Aldabe, Ribeiro, Milosavljevic, \& Bussey, 2012). Because of its anatomical structure, the SIJ mainly experiences rotation along its horizontal axis, where nutation is the posterior rotation of the ilium with respect to the sacrum and counternutation is the opposing motion (Al-Khayer \& Grevitt, 2007).

The SIJ transfers forces between the torso and lower limbs in both static and dynamic situations through form and force closure (Pel, Spoor, Pool-Goudzwaard, Hoek Van Dijke, \& Snijders, 2008; Temme \& Pan, 2017). Form closure occurs through the interlocking of the articular surfaces of the bones, while force closure occurs due to both intrinsically and extrinsically generated ligament tension of the fascial network enclosing the SIJ (Temme \& Pan, 2017). Intrinsic tension can be generated by the motion of the articulating bones stretching ligaments designed to oppose the action, while extrinsic tension is generated through the muscles that attach to and pull on the SIJ (Temme \& Pan, 2017).

As described in the literature, both the intrinsic and extrinsic components of the SIJ are highly innervated. The posterior ligamentous portion of the joint is innervated by the dorsal rami of S1 to S3 and may also be innervated by L4, L5 and S4 as well as the posterior gluteal nerve (Cohen et al., 2013; Hansen et al., 2012). Conversely, the anterior joint capsule of the SIJ is principally supplied by the sacral plexus (Hansen et al., 2012). In regards to the extra-articular SIJ components, testing has shown that the sacrospinous and sacrotuberous ligaments contain proprioceptors, while the dorsal longitudinal SIJ ligament also has nociceptors to sense painful stimuli (Temme \& Pan, 2017).

Even prior to the confirmation of the innervation of the SIJ, its role as a source of low back pain (LBP) was proposed in 1905, but it was overlooked due to the focus on intervertebral discs as pain generators (Al-Khayer \& Grevitt, 2007; Cusi, 2010). To this day, SIJ pain (SIJP) is a broad reaching and poorly defined condition where no single paincausing structure has yet been identified. SIJ dysfunction is thought to be a primary cause, but even that condition is inconsistently defined. Lee et al. (2014) highlighted discrepancies between agreed upon sources of SIJ 
dysfunction, identifying one definition that described SIJP as potentially caused by pelvic asymmetry, hypomobility, hypermobility or instability with or without positional abnormalities. Another quoted source used a stricter definition where SIJP occurs if the joint cannot maintain the self-bracing counternutated position (Lee et al., 2014). Exactly which structures and tissues are aggravated by SIJ dysfunction have not yet been confirmed. Further, no timeline for the development of SIJ dysfunction and the transition to SIJP has been established.

Due to the lack of consensus on the exact development of SIJP, healthcare providers can only confirm that a patient has the condition through a quasi-diagnosis; SIJP is only assigned after the elimination of other pathologies such as spondyloarthropathy or vertebral fractures (Cusi, 2010). The assumed underlying cause of SIJP is that the SIJ is unable to adequately transmit forces between the trunk and lower, leading to pain (Cusi, 2010; Hossain \& Nokes, 2005). The lack of specific characteristics may cause more broad conditions such as non-specific LBP to be assigned as the diagnosis. While $85 \%$ to $90 \%$ of LBP cases are non-specifically diagnosed, estimations suggest that between $10 \%$ and $30 \%$ of LBP cases are chiefly due to SIJP, meaning that the majority of SIJP cases are not properly diagnosed (Adhia, Milosavljevic, Tumilty, \& Bussey, 2016; Eskander et al., 2015). As a result, the exact burden of SIJP on society is not known. However, LBP has been estimated to cost the U.S. government $\$ 50$ billion dollars per year and can impact up to $80 \%$ of adults at some point in their lives (Barbosa, Martins, Barbosa, \& Dos Santos, 2013; Eskander et al., 2015).

The objective of this article is to evaluate the current knowledge surrounding SIJP. An evaluation of the current diagnostic and treatment techniques will allow for conclusions to be drawn on present understanding of the condition. Areas for improvements in research strategies and new areas that still need to be explored will be identified to encourage the development of a more complete picture of SIJP as a pathology.

\section{Clinical Presentation}

\section{Risks}

Risks for SIJP include trauma experienced during events such as motor vehicle accidents and spinal surgeryspecifically lumbar fusion of L4 to S1, which transfers motion and stresses to the SIJ (Cohen et al., 2013; Ledonio, Polly, \& Swiontkowski, 2014; Mason, Chopra, \& Mohanty, 2013). Less acute risks may include true and apparent leg length discrepancy, old age, transitional anatomy such as fused vertebrae, scoliosis, innervation of the SIJ by L5, biomechanical abnormalities, and persistent strain to the joint (Cohen et al., 2013).

Of note, women experience higher rates of SIJP due to increased rates of rheumatoid arthritis and pregnancy (Mason et al., 2013). While pregnancy is associated with higher rates of SIJP, many journal articles refer to the condition as a variation of "pregnancy related posterior pelvic girdle pain syndrome" (Cohen et al., 2013; Mason et al., 2013). Posterior pelvic girdle pain (PPGP) is typically defined as pain that may involve the SIJ, lumbosacral region, and/or pubic symphysis (PS). A lack of definite conclusions around hypermobility of the joint may be due to the structure-specific effects of relaxin. Pearson, Burgess, and Onambélé (2011) saw tendon specific responses to relaxin hormone fluctuations in the knees of normally menstruating women. However, other studies have concluded that while hypermobility and ligament laxity are associated with high relaxin levels, relaxin has not been shown to cause PPGP (Capobianco \& Cher, 2015).

Most studies do not distinguish between women who are 6 months post-partum and nulliparous women. This decision to combine these distinct groups may mask variances in diagnostic and treatment responses between the groups due to differences in SIJP causes. While pregnancy is a risk factor for SIJP, caution should be taken if a study involves post-partum women, as their similarity to idiopathic SIJP groups has not been evaluated. Additionally, articles referring to PPGP should be considered for inclusion in SIJP reviews if the description of the condition matches the set description for SIJP.

\section{Diagnosis}

A healthcare professional will usually arrive at the possibility of SIJP after eliminating all other probable pathologies during their differential diagnosis. These other conditions may include - but are not limited to-sciatica, intervertebral disc ruptures, and stress fractures (George \& Delitto, 2002). The accepted symptoms of SIJP among the literature include pain that occurs below L5 and between the posterior superior iliac spine (PSIS) and the gluteal fold (Temme \& Pan, 2017). More broadly, PPGP, which SIJP is sometimes synonymous with or a subset of, is described as occurring between the posterior iliac crest and gluteal fold and may occur with pain in the PS and posterior thigh (Temme \& Pan, 2017). The most common referral patterns for SIJP involve the buttock and posterolateral thigh (Cohen et al., 2013). Interestingly, elderly patients are more likely to have bilateral pain due to increased arthritic pathologies while younger patients are more likely to have soft tissue problems leading to unilateral pain (Cohen et al., 2013). The individual's daily living may be impacted by a lowered tolerance to standing, walking, sitting, dyspareunia and changes in bladder habits (Cusi, 2010; Temme \& Pan, 2017).

The attending practitioner should be able to reproduce the patient's pain using five to six provocation tests (Table 1) (Temme \& Pan, 2017). SIJP is considered for diagnosis if the patient has 3 positive tests; this diagnostic criterion has $94 \%$ sensitivity and $78 \%$ specificity from previous studies (Cohen et al., 2013). While these levels of sensitivity and specificity are high, they refer only to the ability of the provocation tests to determine individuals who will respond positively to intraarticular (IA) injections. These tests do not allow for discrimination between intra-, peri- and extra-articular sources 
of joint pain. If the patient has 3 positive tests, then they will likely be sent for an IA SIJ injection with a local anesthetic. The IA injection is considered the gold standard for diagnosing SIJP. In particular, dual IA injections, which involve a confirmatory block after the initial injection, have been assigned level II to III evidence, while single blocks only have level III to IV evidence (Simopoulos et al., 2015). Yet these injections, which act as both diagnosis and treatment, can only confirm intra-articular joint pain. The inclusion of peri-articular injections is further discussed in the treatment section of this review.

As knowledge of SIJP, its characteristics, and the contributing anatomical structures continues to grow, new and potentially more specific forms of testing may be incorporated into clinical practice and research. Eskander et al. (2015) tested penny fluoroscopy as a more accurate way of identifying the location of an individual's pain in comparison to currently established provocation testing. Penny fluoroscopies involve marking an individual's pain on a radiograph using a radiopaque object-in this case, an American penny (Eskander et al., 2015). This method of assessment allows for high specificity regarding the exact location of the pain as the researchers set the inclusion cut off at $1 \mathrm{~cm}$ away from the SIJ (as opposed to the more general diagnostic criterion of pain between the PSIS and gluteal fold). However, one large drawback is the radiation exposure required in the technique (Eskander et al., 2015). Of equal concern, while the penny fluoroscopy would allow for very homogenous subject groups to be developed for research purposes, the conclusions drawn from these experiments may not be generalizable to the larger population who have been diagnosed with peri-articular SIJP. As the use of homogenous IA pain groups is already a concern with SIJP studies, this technique should only be used in studies that compare IA to peri-articular pain treatment groups.

Another radiological imaging technique that is currently being explored is X-ray imaging of the PS. Changes in the PS could occur as either a result of direct trauma to the fibrocartilaginous joint or a disruption in the posterior pelvic ring at the SIJ (Becker, Capobianco, \& Seita, 2014). The posterior disruption could drive structural alterations in the PS through changes in compression forces that would be generated by ligamentous tension from sacrum nutation (Becker et al., 2014). Blinded orthopedic surgeons saw changes in the PS x-ray films of $97 \%$ of an SIJP group, but only in 30\% of an LBP group (Becker et al., 2014). Additionally, the SIJP individuals had more visible displacement of the PS while the LBP group was more likely to see osteoarthritic changes of the PS (Becker et al., 2014).

It should be recognized that visual examination of the PS would not discriminate between it being a generator or a marker for SIJP. Of note, Becker et al. (2014) found that 100\% of the SIJP parous females had changes in the PS, while only $22 \%$ of the LBP parous females had changes. However, it is not known whether the mechanism for these changes is the same in both groups, since PS disruption during pregnancy was not reported. One major concern with this as a diagnostic technique is that the patient must be exposed to radiation (Becker et al., 2014). Further studies should be done to confirm whether this test, with additional developed guidelines, is sensitive and specific enough to distinguish SIJP from the general population and from healthy parous females.

Changes in muscle activity are also being sought out as a potential diagnostic technique; however, disagreement on the exact types of changes that occur may prevent their implementation for the foreseeable future. The shift to muscle activation as a method of distinguishing and modeling SIJP occurred because multiple studies found no difference in the absolute range of motion (ROM) between healthy and dysfunctional SIJs (Bussey \& Milosavljevic, 2015). When Bussey and Milosavljevic (2015) measured both SIJ ROM and the electromyography (EMG) for standard core muscles and the biceps femoris muscle, they found that during standing leg raises, the group with positive SIJP had delayed onset of their core muscles with excessive activation of biceps femoris and had increased lumbar flexion velocities. This suggests that impaired feed forward activation of the core muscles may lead to a failure of force closure in the SIJ (Bussey \& Milosavljevic, 2015). The resulting destabilization of the pelvic ring caused feedback activation of the biceps femoris muscle for stabilization, and the combination of the biceps femoris activation and the unstable pelvis drove the increased spinal flexion velocities (Bussey \& Milosavljevic, 2015). Greater onset latencies have been found for muscles that contribute to the stabilization of the SIJ, which include the internal oblique, lumbar multifidus, gluteus maximus, and adductor longus in SIJP (Shadmehr, Jafarian, \& Talebian, 2012). If changes in proper muscle activation patterns for SIJP are standardized, then the use of EMG could be implemented as a more objective form of diagnosis rather than a patient's subjective pain responses to provocation tests.

While EMG provides highly detailed results, it may not be entirely practical in every clinical setting where high volumes of patients require efficient testing. An alternative method is ultrasound, which has been explored as a relatively inexpensive and non-invasive way of measuring muscle activity by looking at changes in muscle thickness during motion testing. Ultrasound of the evaluated transverse abdominis muscle (TrA) for women who either had or did not have PPGP showed no difference in the muscle thickness at rest, but there was a larger increase in thickness for those effected by PPGP during the active straight leg raise (ASLR) (Mens \& Pool-Goudzwaard, 2017). Mens and PoolGoudzwaard (2017) unexpectedly found that the high levels of TrA activation did not help with the pain experienced by the subjects and aggravated it in some cases. However, excessive activation of the TrA may aggravate the long dorsal ligament of the SIJ, which resists TrA induced motion at the SIJ (Pel et al., 2008). Alternatively, this may be due to an issue with the timing of TrA activation (Mens \& Pool-Goudzwaard, 2017). Because EMG was not used, it could not be confirmed whether a change in $\operatorname{TrA}$ onset latency occurred during the experiment. Nevertheless, lack of proper feedforward 
activation of the TrA has been previously documented in SIJP and LBP cases (Marshall \& Murphy, 2006).

Even after many years of research concluding that there were no observable motion differences between healthy and SIJP groups (Cohen et al., 2013), there may be a return to 3D biomechanical motion analysis to further test this idea. Adhia et al. (2016) found that while there were no differences in the net ROMs of individuals with SIJP, there were differences in the ROM trends. Digitized results revealed that individuals with SIJP have a non-linear polynomial trend of motion in the sagittal plane and greater overall motion in the transverse plane as compared with non-SIJP subjects, who only had linear trends in the sagittal plane (Adhia et al., 2016). This may have been due to either ligament stiffness or differences in muscle activation in the SIJP group, such as the increase in the biceps femoris muscle activity that is associated with SIJP (Adhia et al., 2016). While this research is promising in providing a manner of visualizing the condition, its sensitivity and specificity when applied to the general population and inter-tester relatability among healthcare practitioners needs to be evaluated prior to its implementation. While all of these potential diagnostic techniques may be valuable in the future, each study still uses the pain provocation tests to differentiate SIJP from LBP individuals. Future research should endeavor to replicate these studies with an injection based diagnosis to differentiate individuals with SIJP from the general LBP population.

\section{Treatments}

There may be multiple causes of idiopathic SIJP, and in the case of a traumatic injury, the trigger for the pain is often unavoidable. The lack of understanding regarding the development of SIJP in insidious cases results in no single treatment being assigned for individuals afflicted with this condition (Balagué, Mannion, Pellisé, \& Cedraschi, 2012). A patient will begin with conservative therapies and may be prescribed interventional or surgical treatments to manage the chronic SIJP.

\section{Conservative Treatments}

Conservative treatment has been recommended as the primary option for SIJP by the International Association for the Study of Pain (Soisson et al., 2015). The goals of conservative therapy are to return the individual to normal activity through the correction of maladaptive biomechanical imbalances and pain relief (Cohen et al., 2013). However, to date, few if any well designed studies involving conservative treatments have been done, making it difficult to draw strong conclusions about their effectiveness (Cohen et al., 2013).

Manipulation by a manual therapist involves taking joints through passive movements of the ROM and even beyond the elastic barrier through high-velocity, low-amplitude thrusts (Orakifar, Kamali, Pirouzi, \& Jamshidi, 2012). Older studies suggest that the general benefits of manipulation include stretching of the peri-articular soft tissue, improvement of ROM, decreased edema, a reduction in pain, and lessening of muscle spasms (Orakifar et al., 2012). A SIJP intervention study involving low-amplitude SIJ thrusts and low level isotonic muscle contractions over 8 weeks resulted in a reduction in both pain and pelvic angles, which were previously much greater than the angles in the non-SIJP groups (Barbosa et al., 2013). After the treatment, an increase in EMG activity of the rectus femoris as well as the lateral and medial hamstrings was observed (Barbosa et al., 2013). It is not known whether the change in EMG activity was the result of pain relief or led to pain relief, so a repeat of this study with additional testing during the intervention period may be warranted to help determine the exact influence of manipulation.

Manipulation is theorized to cause a decrease in motor neuron activity through inhibitory reflex loops where rapidly stretching agonist muscles leads to the reciprocal inhibition of antagonistic muscles (Orakifar et al., 2012). After applying SIJ thrusts to healthy young women, a drop in the reflex amplitude was observed for up to 20 seconds after the manipulation (Orakifar et al., 2012). Activation of the inhibitory reflex loops may disrupt the pain-spasm-pain cycle, where in this intervention, the activation of the psoas major muscle inhibited the overactive biceps femoris muscle (Orakifar et al., 2012). A different theory suggests that manipulation may take advantage of the large myelinated neurons of mechanoreceptors (Orakifar et al., 2012). The Gate Control Theory explains that large myelinated neurons from mechanoreceptors synapse onto nociceptive neurons via an inhibitory interneuron (Orakifar et al., 2012). In the case of the SIJ, which has been shown to have fewer mechanoreceptors than other joints, there may be less of an inhibitory input onto nociceptors, leading to a hyperalgesic state for chronic SIJP (Orakifar et al., 2012). Manipulation may be able to reduce this hyperalgesia by activating the few mechanoreceptors that are present. It seems likely that the pain relief experienced by the subjects may have been a combination of both reflexinduced changes in muscle activation and the Gate Control Theory of Pain (Orakifar et al., 2012).

While these results are positive regarding the effects of manipulation, whether the effect can experience temporal summation needs to be studied to better develop useful treatment plans. To date, while studies of various methodological qualities have been done, only poorly controlled studies have shown evidence that manipulation works (Cohen et al., 2013), so well-designed studies need to be done on the process of manipulation to determine if they are truly worth the monetary investment. Observations from these new research areas may lead to the invention of more focused treatments that exploit these novel pain relieving mechanisms. However, despite the concerns surrounding research quality, the low risk associated with manipulation does lead to the suggestion that it should be considered for use by trained healthcare professionals (Cohen et al., 2013).

Motor control therapies attempt to target and improve the failure of force closure by re-establishing the feedforward activation and strength of stabilizing muscles while reducing instability feedback activation. SIJP individuals can have 
increased descent of their pelvic floor and increased excursion of the diaphragm during ASLR testing (O'Sullivan \& Beales, 2007). A motor learning therapy involving the pelvic floor over 12 weeks, with an initial 3 weeks using a pelvic belt, saw an increase in the activation of the pelvic floor which correlated with improvements in ASLR responses and pain scores (O'Sullivan \& Beales, 2007). This result, along with observed improvements in the subjects' continence, suggests that a weakness in the pelvic floor may contribute to the development or be the result of SIJP (O'Sullivan \& Beales, 2007).

More recently, Sjödahl, Gutke, Ghaffari, Strömberg, and Öberg (2016) measured the timing of pelvic floor descent during ASLR for women with pregnancy related PPGP and for healthy groups. They found that there was a delay in the activation of both the pelvic floor muscles and lower back muscles as well as a depression in the pelvic floor in the PPGP group (Sjödahl et al., 2016). This finding supports the feedforward activation impairment theory in SIJP and confirms that the pelvic floor muscle is implicated in creating force closure. Based on these more recent results, it is recommended that the O'Sullivan and Beales (2007) experiment should be repeated with measurements of the activation time of the pelvic floor to see if a motor intervention treatment strategy improves both ASLR scores and function.

One potential treatment that individuals with SIJP can use in their daily lives is a pelvic belt. The belts provide joint compression and increase stiffness, allowing for greater joint stability (Beales, O'Sullivan, \& Briffa, 2010; Temme \& Pan, 2017). While many patients with SIJP say that the belt does provide pain relief, this improvement seems counterintuitive as sacral joint compression is used as a pain provocation test (Beales et al., 2010). However, since there is no single pain provocation test for SIJP, those who find relief using the belts may not have positive compression tests. Additionally, the belt may provide much wider dispersion of compression and therefore less localized compressive stress

Hammer et al. (2015) tested the use of pelvic belts on MRI confirmed non-inflammatory SIJP populations with age matched controls and saw improvements in pain scores after six weeks that were comparable to surgical fusion treatment. Additionally, rectus femoris muscle activity was decreased with moderate application and there were visible improvements in cadence and gait velocity (Hammer et al., 2015). This decrease in rectus femoris muscle activity opposes results mentioned prior where manipulation caused an increase in activity (Barbosa et al., 2013). Neither study commented on the timing of this activation before or after the interventions, which makes a direct comparison difficult and thus the results of both studies should be interpreted carefully. Evaluation of immediate responses to the belt in injection controlled SIJP groups for MRI pelvic morphometry and EMG during single leg stance showed diverging results (Soisson et al., 2015). There appeared to be either a significant positive effect or a significant negative effect of using the belt - suggesting there may be responders and non-responders to pelvic belts, which has also been seen in earlier studies
(Soisson et al., 2015). Overall, there was no significant change in the combined EMG patterns of the SIJP group with the addition of the pelvic belts; this may be due to the single leg stance, which causes counternutation and a more stable joint position (Soisson et al., 2015).

Pelvic belts increased gluteus maximus muscle activation while slowing the onset time and reducing the activation of the biceps femoris muscle (Jung, Jeon, Oh, \& Kwon, 2013). The belts likely unloaded sensitized structures such as the sacroiliac ligaments and provided proprioceptive feedback that the joint was stable (Jung et al., 2013). A reduction in the biceps activation may have improved the gluteus maximus muscle activation since biceps activation can inhibit gluteus maximus activation, consequently weakening it and feeding back into the lack of stability (Jung et al., 2013).

Conservative treatments have the potential to treat SIJP in non-invasive manners. The ability of manipulation and motor control interventions to impact impaired feed forward activation needs to be assessed further with additional EMG studies. The temporal effects of each intervention must be evaluated for long term validity since many of the manipulation studies involved a single intervention session, or did not track improvements throughout the course of a longterm intervention. Also, alternative therapies for those who respond negatively to pelvic belts should be researched for their efficacy. It should be determined if a positive sacral compression test warrants hesitation from healthcare professionals regarding the prescription of a pelvic belt for managing SIJP.

\section{Interventional Treatments}

Oral medications are a systemic treatment that can target multiple sources of pain, which is advantageous with SIJP where a diverse number of tissues are involved. Antiinflammatories can be prescribed for potential underlying causes such as spondyloarthropathies and osteoarthritis (Duruöz, Turan, Gürgan, \& Deveci, 2012). Opioids may also be prescribed for general pain relief, but healthcare professionals recommend a reduction in opioid use due to studies suggesting that long term use is linked with worsening pain and increased disability scores (Zgierska et al., 2016).

SIJ injections are a more localized treatment option offered to patients, with the most widely used being a guided IA injection containing a combination of a local anesthetic and a steroid. The anesthetic component of the serum targets nociceptors, which have been found in the joint capsule, ligaments and subchondral bone (Szadek, Hoogland, Zuurmond, De Lange, \& Perez, 2010). While IA injections are the gold standard for diagnosis, they have only been assigned level IV evidence for their therapeutic use and have been found to improve pain in $18 \%$ to $57 \%$ of subjects depending on the study being consulted (Borowsky \& Fagen, 2008; Cusi, 2010; Simopoulos et al., 2015).

A combination of IA and peri-articular injections has been found to improve pain reduction results when compared to IA only injection protocols (Borowsky \& Fagen, 2008). 
Peri-articular injections operate based on the assumption that the joint itself may not be the primary pain source, but it is instead the surrounding nerves and ligaments causing pain. Palsson et al. (2015) found that induction of pain in the long posterior sacroiliac ligament altered activity in the pelvic girdle stabilizing muscles in a way that resembled trends seen in SIJP patients. The iliolumbar ligament may also act as a peri-articular pain generator in individuals with an excessively flexed lumbar spine that places a high load on the ligament (Pel et al., 2008). Further, hypermobility in pregnancy may contribute to pain from the pelvic ligaments and joint capsule in women with pregnancy related PPGP (Al-Khayer \& Grevitt, 2007; Walker, 1992). However, contradictory results from studies suggest that peri-articular injections do not work as well in pregnancy related PPGP patients when compared with SIJP patients (Capobianco \& Cher, 2015). This may be due to the involvement of other structures such as the PS as the extra-articular sources of pregnancy related pain, which are not included in peri-articular injections.

In the United States, despite only a growth of $18 \%$ in the individuals receiving Medicare coverage, there has been an increase of $331 \%$ in Medicare covered injection rates (Manchikanti, Hansen, Pampati, \& Falco, 2014). This highlights a dramatic increase in SIJ injection rates. One main concern with this is that other injections, such as for facet joints, have had more restrictive guidelines placed on them to ensure their proper use, which has yet to occur for SIJ injections (Manchikanti et al., 2014). While it is a valid point that SIJ injections are not as controlled as facet injections, the Medicare study did not separate the use of injections into diagnostic and therapeutic categories, whereas the facet joint injection statistics were associated with therapeutic roles (Manchikanti et al., 2014). The breakdown of SIJ injection use would be valuable information to elucidate if the injections are being used primarily as a diagnostic technique or if there is a select group of individuals who are benefitting from the therapeutic use. If the rate of diagnostic use is high, this may be due to either a lack of sensitivity and specificity in the pain provocation testing or a lack of effectiveness on the part of SIJ injections in pain management. New imaging techniques that are sensitive to inflammation such as single emission CT or radionucleotide bone scans may help to determine if any inflammation is actually occurring within the joint, and an IA injection may assist in providing objective evidence for performing SIJ injections (Cohen et al., 2013). While combined injections are marginally better than IA only injections, there are no standardized procedures for periarticular injections (Hansen et al., 2012). Variation in how many structures are included in the injection protocol makes comparisons between studies difficult.

Prolotherapy is an alternative to the standard steroid and anesthetic serum, and involves either the injection of an inert irritating substance or platelet-rich plasma with the goal of triggering an inflammatory response to repair damaged tissue (Cohen et al., 2013). When the deep interosseous ligament of the SIJ was peri-articularly injected with Narapin and glucose over multiple sites, improvements were seen in pain relief and strength (Mitchell, Rose, \& Barnard, 2014). Moreover, a randomized study saw better long term positive outcomes for those who received a dextrose IA injection versus steroid injections (Cohen et al., 2013). A previous study showed that prolotherapy injections are more effective than blinded steroid injections at 3 months post-injection (Hansen et al., 2012).

Overall, positive responses to injections tend to increase with age and a presentation of bilateral pain (Kennedy et al., 2015). These factors are likely related through the increase in arthritis related IA pain (Kennedy et al., 2015). Positive responses to IA injections for other inflammatory pathologies have been seen with individuals who have ankylosing spondyloarthropathy (Kennedy et al., 2015). It has been suggested that the effectiveness of injections - particularly IA ones - may be limited by the joint space, as it only contains between $1 \mathrm{ml}$ to $2 \mathrm{ml}$ of volume, making complete anesthetization of the aggravating structures difficult (Kennedy et al., 2015). These injections also have potential complications such as increased pain, vasovagal reactions, pyogenic sacroiliitis, temporary sciatic palsy, and plastic changes in the peripheral nervous system with continuous application (Hansen et al., 2012; Kennedy et al., 2015). Sciatic palsy may only be a risk for individuals with anterior capsule tears which would allow the anesthetic to come into contact with the sacral plexus by diffusing out of the joint space (Kennedy et al., 2015).

Besides treating inflammatory causes of SIJP, the main value of IA injections may be as a prognostic tool for success of radiofrequency ablations (Hansen et al., 2012). Radiofrequency ablations, which have been used to treat spinal pain since the 1970s, involve the creation of lesions in the lateral nerves as a minimally invasive procedure (Cohen et al., 2013). There are three main types of ablation: conventional, cooled and pulsed. Previous research suggested that only cooled had fair evidence in comparison to pulsed and conventional, but a more recent study suggested that cooled and conventional had similar outcomes except for a greater risk of steroid use with the cooled technique (Cheng, Pope, Dalton, Cheng, \& Bensitel, 2013; Hansen et al., 2012). Complications of this treatment include post-procedural numbness, tingling and medial branch neuritis (Cohen et al., 2013). Seemingly contrary to the initial statement that IA injections are good for selecting individuals for ablation, these procedures typically have better success in younger patients and appear to be linked with successful extra-articular injections, while regular opioid use and old age, which correlate with positive responses to IA injections, are associated with treatment failure (Cohen et al., 2013). An up to date systematic review of the subgroups of SIJP patients who respond best to radiofrequency ablation would serve healthcare professionals in helping to determine which patients should be offered this treatment.

\section{Surgical Treatments}

Surgical interventions are only recommended for those who have failed to respond to conservative treatment 
(Hammer et al., 2015). With its first reported use in 1921, arthrodesis involves the fusion of the sacrum and ilium through the placement of surgical implants (Capobianco \& Cher, 2015). The main goal of the surgery is to reduce the motion in the joint and thus prevent aggravation of problematic structures. This procedure works well in women who experience pregnancy related PPGP as they see a significant improvement in pain, function and quality of life (Capobianco \& Cher, 2015). These results may be due to the correction of the destabilization of the pelvic ring through PS disruption; however, results for SIJP with unspecified causes are less convincing.

Initial results from a study comparing conservative treatment with surgical implants showed that the surgical group had significant improvements in function when compared to the conservative treatment group who saw no clinical improvement (Dengler et al., 2017). The conservative treatment group was offered the opportunity to cross over to the surgical intervention after a review of the initial results (Dengler et al., 2017). Those who crossed over saw similar improvements in function to the original surgical group. It should be noted that the primary funding source for this study was the company SI-BONE who provided the surgical implants (Dengler et al., 2017). This same company also employed many of the researchers and compiled the published statistics for the results internally prior to being approved by the research team (Dengler et al., 2017).

The optimistic results of the Dengler et al. (2017) study have not been achieved by other articles. Contrasting results from an older study showed an $82 \%$ dissatisfaction rate with the operation and a reoperation rate of $65 \%$ (Cohen et al., 2013). While this may be the result of gross surgical error, fusion is only strongly recommended for those with unstable pelvic ring injuries, and those with other causes should be considered carefully (Mason et al., 2013). Mason et al. (2013) found that patients who had prior spinal surgeries had worse outcomes within the three-year sacroiliac joint post-operative period than those who had not previously had surgery. It was suggested that the SIJP had not been relieved, but the nature of the remaining pain had shifted due to the new procedure transferring stresses on to new structures that had not been previously implicated (Mason et al., 2013).

Overall, multiple studies have shown success rateswhere the surgery improved pain and function scores - of $70 \%$ with SIJ fusion (Cohen et al., 2013). Despite this respectable success rate, the recommended qualification criteria from the North American Spine Society for insurance companies are extremely restrictive in determining who can access the procedure (Simopoulos et al., 2015). In order to qualify, an individual must meet all of the following: "failed conservative management, greater than 3 pain provocation tests, nonradiating unilateral pain, localized tenderness, negative diagnostic imaging pain results, greater than $75 \%$ to $80 \%$ pain reduction with controlled diagnostic blocks and absence of generalized pain behavior" (Simopoulos et al., 2015). To best benefit those who require surgical treatment, more lenient qualification criteria should be compared with the current restrictions. The characteristics of those who find the surgical treatment to be successful should be evaluated by independent researchers so that appropriate recommendations for treatments can be made.

\section{Conclusion}

There is still much that is unknown regarding SIJP. A model of the development and manifestations of SIJP has yet to be developed in either humans or animals. If SIJP can be modeled, then the existence of sub-classifications, such as IA, extra-articular and post-partum, can be determined using physiological evidence from the sensory-motor system. Understanding how the SIJP manifests for each group will allow for both the improvement in current diagnostic techniques and the addition of new techniques that can reliably diagnose peri- and extra-articular pain. The inclusion of those with non-IA pain will allow for new comparisons to be made on treatment use in a group that had previously been ignored. Additional research into the underlying exact mechanisms for conservative therapies may indicate which subcategory of individuals will best respond to the treatments. This new research would not only determine treatment efficacy overall, but it would also help to increase patient flow by improving practitioner decision making as to which treatment will be most effective.

\section{Acknowledgements}

The authors of this study have no conflicts of interest to report. Special thanks to Lisa Philps for assisting with the photography for this review.

\section{References}

Adhia, D. B., Milosavljevic, S., Tumilty, S., \& Bussey, M. D. (2016). Innominate movement patterns, rotation trends and range of motion in individuals with low back pain of sacroiliac joint origin. Manual Therapy, 21, 100-108. doi:10.1016/j.math.2015.06.004

Al-Khayer, A., \& Grevitt, M. P. (2007). The sacroiliac joint: An underestimated cause for low back pain. Journal of Back and Musculoskeletal Rehabilitation, 20, 135-141. doi:10.3233/BMR-2007-20401

Aldabe, D., Ribeiro, D. C., Milosavljevic, S., \& Bussey, M. D. (2012). Pregnancy-related pelvic girdle pain and its relationship with relaxin levels during pregnancy: A systematic review. European Spine Journal, 21(9), 1769-1776. doi:10.1007/s00586-012-2162-x

Balagué, F., Mannion, A. F., Pellisé, F., \& Cedraschi, C. (2012). Non-specific low back pain. Lancet, 379(9814), 482-91. doi:10.1016/S01406736(11)60610-7 
Barbosa, A. C., Martins, F. L. M., Barbosa, M. C. S. A., \& Dos Santos, R. T. (2013). Manipulation and selective exercises decrease pelvic anteversion and low-back pain: A pilot study. Journal of Back and Musculoskeletal Rehabilitation, 26(1), 33-36. doi:10.3233/BMR-2012-0347

Beales, D. J., O’Sullivan, P. B., \& Briffa, N. K. (2010). The effects of manual pelvic compression on trunk motor control during an active straight leg raise in chronic pelvic girdle pain subjects. Manual Therapy, 15(2), 190-199. doi:10.1016/j.math.2009.10.008

Becker, S., Capobianco, R., \& Seita, M. (2014). Is sacroiliac joint pain associated with changes in the pubic symphysis? A radiographic pilot study. European Journal of Orthopaedic Surgery \& Traumatology, 25. doi:10.1007/s00590-014-1575-0

Borowsky, C. D., \& Fagen, G. (2008). Sources of sacroiliac region pain: Insights gained from a study comparing standard intra-articular injection with a technique combining intra- and peri-articular injection. Archives of Physical Medicine and Rehabilitation, 89(11), 2048-2056. doi:10.1016/j.apmr.2008.06.006

Bussey, M. D., \& Milosavljevic, S. (2015). Asymmetric pelvic bracing and altered kinematics in patients with posterior pelvic pain who present with postural muscle delay. Clinical Biomechanics, 30(1), 71-77. doi:10.1016/j.clinbiomech.2014.11.002

Capobianco, R., \& Cher, D. (2015). Safety and effectiveness of minimally invasive sacroiliac joint fusion in women with persistent post-partum posterior pelvic girdle pain: 12-month outcomes from a prospective, multicenter trial. SpringerPlus, 4(1), 570. doi:10.1186/s40064-015-1359-y

Cheng, J., Pope, J. E., Dalton, J. E., Cheng, O., \& Bensitel, A. (2013). Comparative outcomes of cooled versus traditional radiofrequency ablation of the lateral branches for sacroiliac joint pain. The Clinical Journal of Pain, 29(2), 132-137. doi:10.1097/AJP.0b013e3182490a17

Cohen, S. P., Chen, Y., \& Neufeld, N. J. (2013). Sacroiliac joint pain: a comprehensive review of epidemiology, diagnosis and treatment. Expert Review of Neurotherapeutics, 13(1), 99-116. doi:10.1586/ern.12.148

Cusi, M. F. (2010). Paradigm for assessment and treatment of SIJ mechanical dysfunction. Journal of Bodywork and Movement Therapies, 14(2), 152-161. doi:10.1016/j.jbmt.2009.12.004
Dengler, J. D., Kools, D., Pflugmacher, R., Gasbarrini, A., Prestamburgo, D., Gaetani, P., ... Sturesson, B. (2017). 1 -Year results of a randomized controlled trial of conservative management vs. minimally invasive surgical treatment for sacroiliac joint pain. Pain Physician, 20(6), 537-550. Retrieved from http://www.ncbi.nlm.nih.gov/pubmed/28934785

Duruöz, M. T., Turan, Y., Gürgan, A., \& Deveci, H. (2012). Evaluation of metabolic syndrome in patients with chronic low back pain. Rheumatology International, 32(3), 663-667. doi:10.1007/s00296-010-1693-x

Eskander, J. P., Ripoll, J. G., Calixto, F., Beakley, B. D., Baker, J. T., Healy, P. J., ... Sharma, S. (2015). Value of examination under fluoroscopy for the assessment of sacroiliac joint dysfunction. Pain Physician, 18(5), E781-6. Retrieved from http:/www.painphysicianjournal.com/current/pdf?artic le $=\mathrm{MjQwOQ}==\% 5 \mathrm{Cnhttp}$ ://ovidsp.ovid.com/ovidweb. cgi? $=\mathrm{JS} \& \mathrm{PAGE}=$ reference $\& \mathrm{D}=$ emed $13 \& \mathrm{NEWS}=\mathrm{N}$ \&AN=2015396637\%5Cnhttp://ovidsp.ovid.com/ovidw eb.cgi? $\mathrm{T}=\mathrm{JS} \& \mathrm{PAGE}=$ reference $\& \mathrm{D}=$ prem $\& N E W S=\mathrm{N}$ $\& A N=26431131$

George, S. Z., \& Delitto, A. (2002). Management of the athlete with low back pain. Clinics in Sports Medicine, 21(1), 105-120. doi:10.1016/S0278-5919(03)00060-7

Hammer, N., Möbius, R., Schleifenbaum, S., Hammer, K.H., Klima, S., Lange, J. S., ... Milani, T. L. (2015). Pelvic belt effects on health outcomes and functional parameters of patients with sacroiliac joint pain. Plos One, 10(8), e0136375. doi:10.1371/journal.pone.0136375

Hansen, H., Manchikanti, L., Simopoulos, T. T., Christo, P. J., Gupta, S., Smith, H. S., ... Cohen, S. P. (2012). A systematic evaluation of the therapeutic effectiveness of sacroiliac joint interventions. Pain Physician, 15(3), E247-78. Retrieved from http://www.ncbi.nlm.nih.gov/pubmed/22622913

Hossain, M., \& Nokes, L. D. M. (2005). A model of dynamic sacro-iliac joint instability from malrecruitment of gluteus maximus and biceps femoris muscles resulting in low back pain. Medical Hypotheses, 65(2), 278-281. doi:10.1016/j.mehy.2005.02.035

Jung, H. S., Jeon, H. S., Oh, D. W., \& Kwon, O. Y. (2013). Effect of the pelvic compression belt on the hip extensor activation patterns of sacroiliac joint pain patients during one-leg standing: A pilot study. Manual Therapy, 18(2), 143-148. doi:10.1016/j.math.2012.09.003 
Kennedy, D. J., Engel, A., Kreiner, D. S., Nampiaparampil, D., Duszynski, B., \& Macvicar, J. (2015).

Fluoroscopically Guided Diagnostic and Therapeutic Intra-Articular Sacroiliac Joint Injections: A Systematic Review. Pain Medicine (United States), 16(8), 1500-1518. doi:10.1111/pme.12833

Kim, Y. H., Yao, Z., Kim, K., \& Park, W. M. (2014). Quantitative investigation of ligament strains during physical tests for sacroiliac joint pain using finite element analysis. Manual Therapy, 19(3), 235-241. doi:10.1016/j.math.2013.11.003

Ledonio, C. G. T., Polly, D. W., \& Swiontkowski, M. F. (2014). Minimally invasive versus open sacroiliac joint fusion: Are they similarly safe and effective? Clinical Orthopaedics and Related Research, 472(6), 18311838. doi:10.1007/s11999-014-3499-8

Lee, J. H., Yoo, W. G., Kim, M. H., Oh, J. S., Lee, K. S., \& Han, J. T. (2014). Effect of posterior pelvic tilt taping in women with sacroiliac joint pain during active straight leg raising who habitually wore high-heeled shoes: A preliminary study. Journal of Manipulative and Physiological Therapeutics, 37(4), 260-268. doi:10.1016/j.jmpt.2014.01.005

Manchikanti, L., Hansen, H., Pampati, V., Falco, F.J.E. (2014). Percutaneous adhesiolysis procedures in the medicare population: Analysis of utilization and growth patterns from 2000 to 2011. Pain Physician, 17(2), E129-E139. Retrieved from http://www.embase.com/search/results?subaction=vie wrecord\&from=export\&id=L372671026

Marshall, P., \& Murphy, B. (2006). The effect of sacroiliac joint manipulation on feed-forward activation times of the deep abdominal musculature. Journal of Manipulative and Physiological Therapeutics, 29(3), 196-202. doi:10.1016/j.jmpt.2006.01.010

Mason, L. W., Chopra, I., \& Mohanty, K. (2013). The percutaneous stabilization of the sacroiliac joint with hollow modular anchorage screws: A prospective outcome study. European Spine Journal, 22(10), 2325-2331. doi:10.1007/s00586-013-2825-2

Mens, J. M. A., \& Pool-Goudzwaard, A. (2017). The transverse abdominal muscle is excessively active during active straight leg raising in pregnancy-related posterior pelvic girdle pain: an observational study. BMC Musculoskeletal Disorders, 18(1), 372. doi:10.1186/s12891-017-1732-9

Mitchell, B., Rose, R., \& Barnard, A. (2014). Prolotherapy for sacroiliac joint pain-12 months outcomes. Journal of Science and Medicine in Sport, 18. doi:

10.1016/j.jsams.2014.11.351

O’Sullivan, P. B., \& Beales, D. J. (2007). Changes in pelvic floor and diaphragm kinematics and respiratory patterns in subjects with sacroiliac joint pain following a motor learning intervention: A case series. Manual Therapy, 12(3), 209-218. doi:10.1016/j.math.2006.06.006

Orakifar, N., Kamali, F., Pirouzi, S., \& Jamshidi, F. (2012). Sacroiliac joint manipulation attenuates alphamotoneuron activity in healthy women: A quasiexperimental study. Archives of Physical Medicine and Rehabilitation, 93(1), 56-61.

doi:10.1016/j.apmr.2011.05.027

Palsson, T.S., Hirata, R.P., Graven-Nielsen, T. (2015). Experimental pelvic pain impairs the performance during the active straight leg raise test and causes excessive muscle stabilization. Clinical Journal of Pain, 31, 642-651. doi:10.1097/AJP.0000000000000139

Pearson, S. J., Burgess, K. E., \& Onambélé, G. L. (2011). Serum relaxin levels affect the in vivo properties of some but not all tendons in normally menstruating young women. Experimental Physiology, 96(7), 681688. doi:10.1113/expphysiol.2011.057877

Pel, J. J. M., Spoor, C. W., Pool-Goudzwaard, A. L., Hoek Van Dijke, G. A., \& Snijders, C. J. (2008). Biomechanical analysis of reducing sacroiliac joint shear load by optimization of pelvic muscle and ligament forces. Annals of Biomedical Engineering, 36(3), 415-424. doi:10.1007/s10439-007-9385-8

Shadmehr, A., Jafarian, Z., \& Talebian, S. (2012). Changes in recruitment of pelvic stabilizer muscles in people with and without sacroiliac joint pain during the active straight-leg-raise test. Journal of Back and Musculoskeletal Rehabilitation, 25(1), 27-32. doi:10.3233/BMR-2012-0307

Simopoulos, T. T., Manchikanti, L., Gupta, S., Aydin, S. M., Kim, C. H., Solanki, D., ... Hirsch, J. A. (2015). Systematic review of the diagnostic accuracy and therapeutic effectiveness of sacroiliac joint interventions. Pain Physician, 18(5), E713-E756. Retrieved from http://www.scopus.com/inward/record.url?eid=2-s2.084942093986\&partnerID=tZOtx3y1

Sjödahl, J., Gutke, A., Ghaffari, G., Strömberg, T., \& Öberg, B. (2016). Response of the muscles in the pelvic floor and the lower lateral abdominal wall during the Active 
Straight Leg Raise in women with and without pelvic girdle pain: An experimental study. Clinical

Biomechanics, 35, 49-55.

doi:10.1016/j.clinbiomech.2016.04.007

Soisson, O., Lube, J., Germano, A., Hammer, K. H., Josten, C., Sichting, F., ... Hammer, N. (2015). Pelvic belt effects on pelvic morphometry, muscle activity and body balance in patients with sacroiliac joint dysfunction. PLOS ONE, 10(3), 1-27. doi:10.1371/journal.pone.0116739

Szadek, K. M., Hoogland, P. V. J. M., Zuurmond, W. W. A., De Lange, J. J., \& Perez, R. S. G. M. (2010). Possible nociceptive structures in the sacroiliac joint cartilage: An immunohistochemical study. Clinical Anatomy, 23(2), 192-198. doi:10.1002/ca.20908

Temme, K. E., \& Pan, J. (2017). Musculoskeletal approach to pelvic pain. Physical Medicine and Rehabilitation Clinics of North America.

doi:10.1016/j.pmr.2017.03.014

Walker, J. M. (1992). The sacroiliac joint: a critical review. Physical Therapy, 72(12), 903-916. Retrieved from http://link.galegroup.com.subzero.lib.uoguelph.ca/apps $/$ doc/A!3309995/AONE?u=guel77241\&sid=AONE\&xi $\mathrm{d}=5290 \mathrm{~d} 856$

Zgierska, A. E., Burzinski, C. A., Cox, J., Kloke, J., Stegner, A., Cook, D. B., ... Bačkonja, M. (2016). Mindfulness meditation and cognitive behavioral therapy intervention reduces pain severity and sensitivity in opioid-treated chronic low back pain: Pilot findings from a randomized controlled trial. Pain Medicine, 17(10), 1865-1881. doi:10.1093/pm/pnw006 


\section{Tables and Figures}

Table 1. Pain Provocation Tests for Sacroiliac Joint Pain

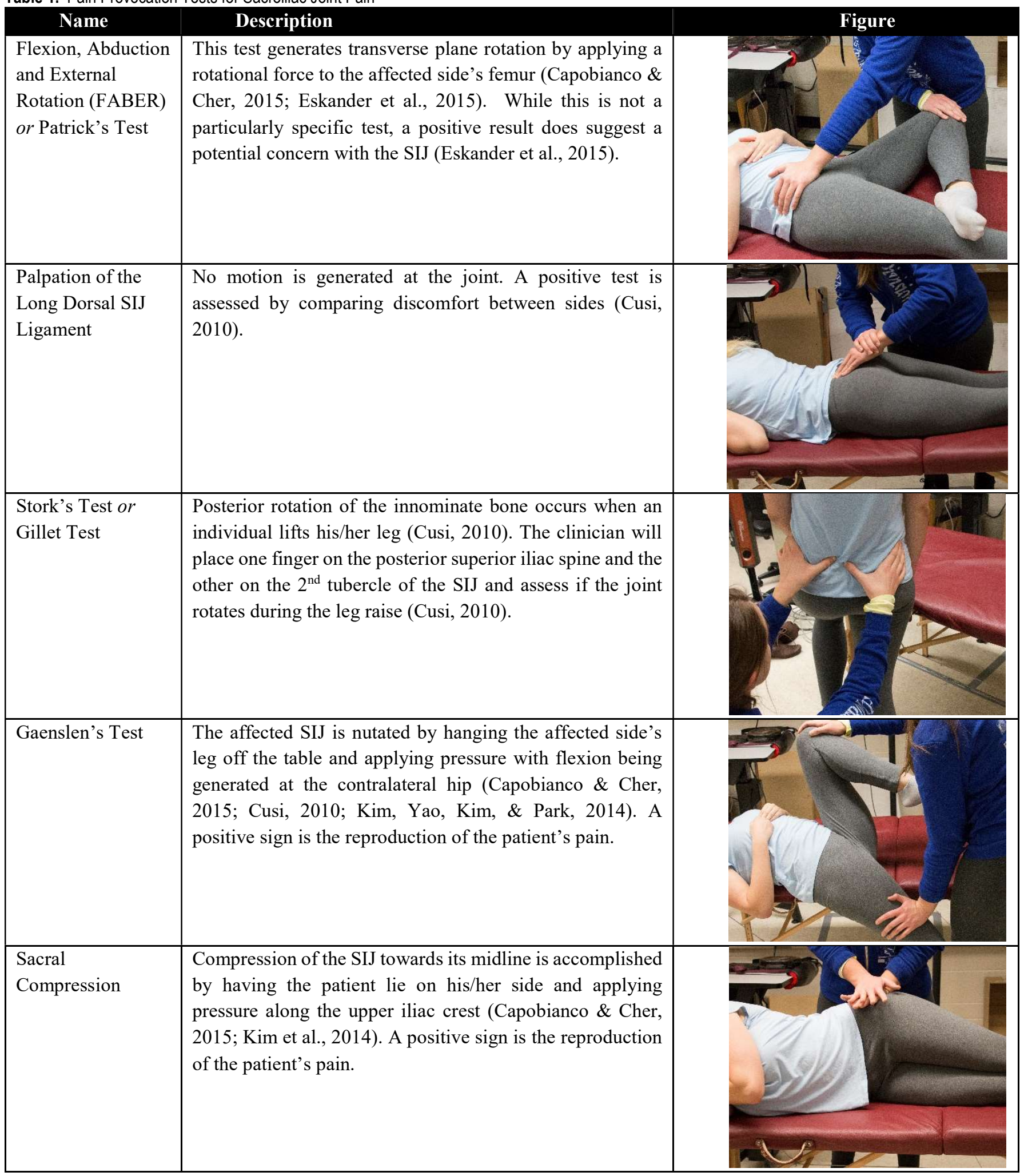




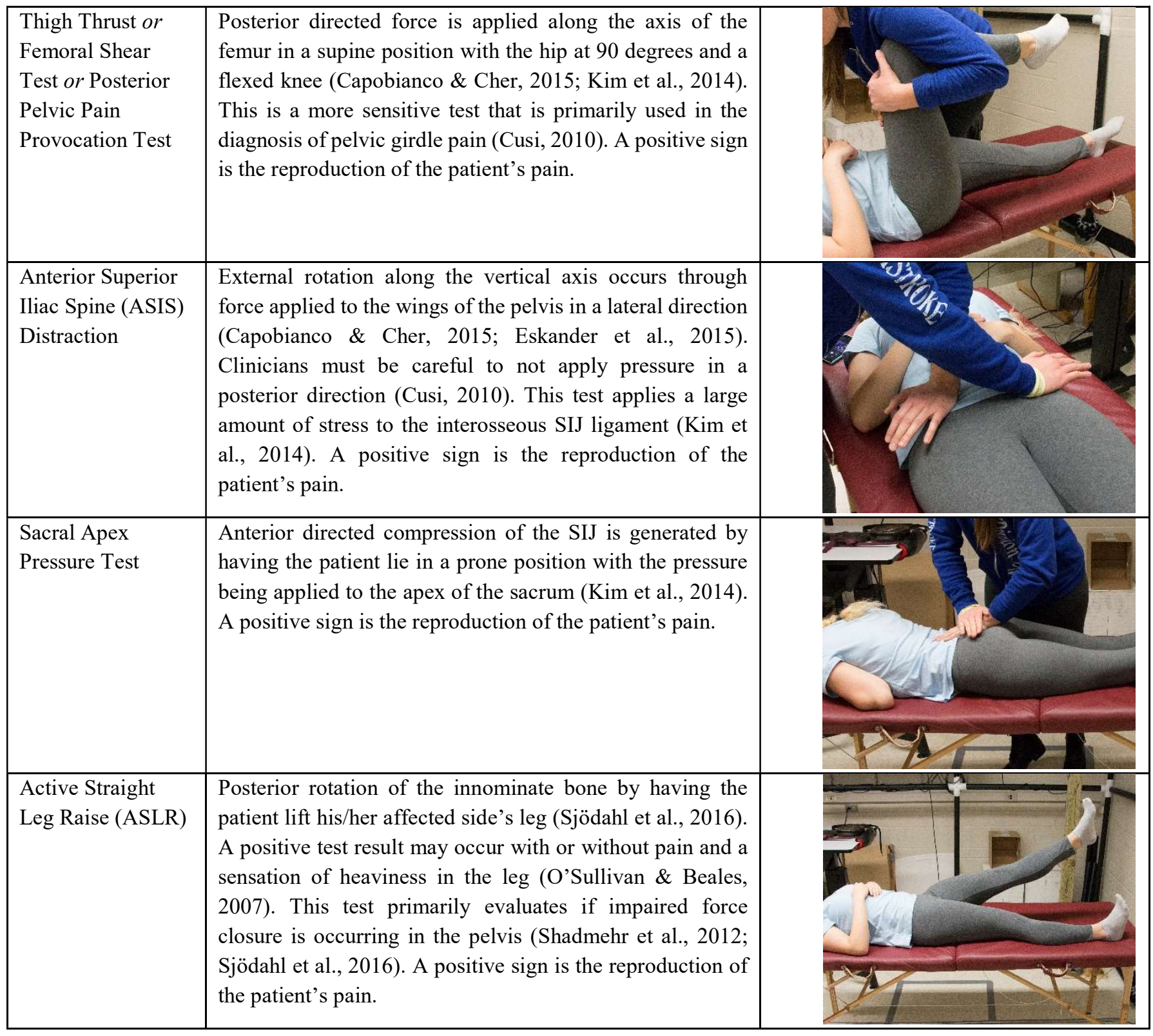

